\title{
Crystallographic Analysis of Analogous Silicon- and Carbon-Containing Di(Cyanate Ester)s and Tri(Cyanate Ester)s
}

$\underline{\text { Kamran B. Ghiassi }}{ }^{1}$, Andrew J. Guenthner ${ }^{1}$, Sean M. Ramirez ${ }^{2}$, Michael D. Ford ${ }^{2}$, Denisse Soto ${ }^{3}$, Jerry A. Boatz ${ }^{1}$, and Joseph M. Mabry ${ }^{1}$

${ }^{1}$ Air Force Research Laboratory, Aerospace Systems Directorate, Edwards AFB, California 93524, United States

${ }^{2}$ ERC Incorporated, Edwards AFB, California 93524, United States

${ }^{3}$ Naval Surface Warfare Center Indian Head EOD Technology Division, Indian Head, Maryland 20640, United States

Cyanate esters are versatile, thermosetting monomers that are commercially important due to their outstanding physical properties such as fire and heat resistivity. This class of molecules also possesses relatively low melting points, allowing for improved processability and manufacturing. While studies of this class of polymers are prominent, the monomers tend to shy away from focus. In an effort to understand organic crystal engineering of these compounds, four structures of silicon- and carbon-containing di(cyanate ester)s and tri(cyanate ester)s are presented and analyzed using single-crystal X-ray diffraction. This data, in concert with extensive thermodynamic and computational studies, assist in understanding how a subtle change, such as exchanging quaternary central carbon for a silicon, affects the molecular degrees of freedom. From a crystallographic standpoint, this affects the packing efficiency and intermolecular interactions. 